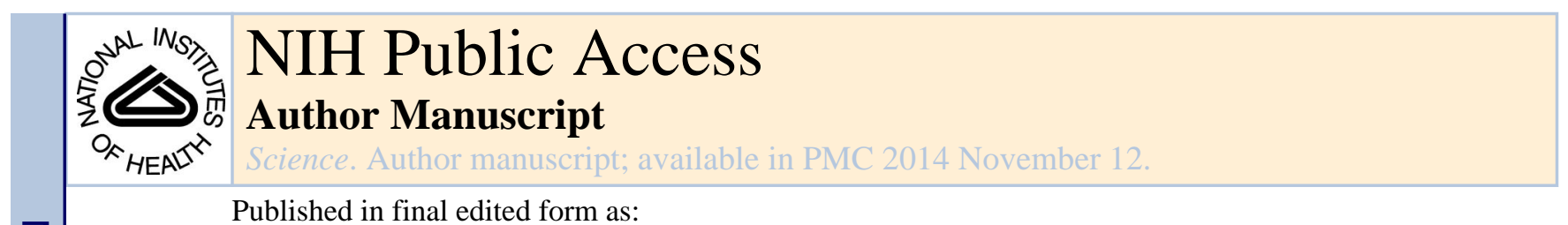

Published in final edited form as:

Science. 2013 October 4; 342(6154): 47-49. doi:10.1126/science.1244492.

\title{
Social and biological contagions
}

Chris T. Bauch ${ }^{1}$ and Alison P. Galvani ${ }^{2,{ }^{*}}$

${ }^{1}$ Department of Applied Mathematics, University of Waterloo, Waterloo, ON N2L 3G1, Canada

${ }^{2}$ School of Public Health, Yale University, New Haven, CT 06520, USA

\section{Abstract}

The coupling of social and biological contagion in human populations can have positive or negative outcomes.

Despite the invention of control measures like vaccines, infectious diseases remain part of human existence. Ideas, sentiments or information can also be contagious $(1,2)$. Such social contagion is akin to biological contagion-both spread through a replication process that is blind to the consequences for the individual, and if each person transmits to more than one person, the explosive power of exponential growth creates an epidemic. Social contagions may cause irrational "fever". Isaac Newton, having lost $£ 20,000$ in the speculative South Sea Bubble, commented that he could "calculate the movement of the stars, but not the madness of men" (3). Systems where both types of contagion are coupled to one anotheran infectious disease spreading by biological contagion and a social contagion concerning the disease-offer unique scientific challenges and are increasingly important for public health (4-15).

Social contagions can exhibit complex dynamics, such as the tipping points and cascading instabilities observed in financial collapse (2). Social contagion can be conceptualized as a network, where each node is a person, and the network links are social contacts through which the contagion can spread (see the figure, panel A). Infectious diseases can also exhibit complex dynamics and be conceptualized as a network through which the biological contagion spreads (see the figure, panel B).

When a social contagion is coupled to a biological contagion, the resulting disease-behavior system can exhibit dynamics that do not occur when the two subsystems are uncoupled and in isolation from one another. This illustrates the lesson of complexity science that the whole is more than the sum of the parts (see the figure, panel C).

For example, high levels of pediatric vaccine coverage can decrease disease incidence to very low levels, reducing the perceived danger of infection and hence the urgency to get vaccinated. Subsequently, if a highly connected node in the social contact network (such as celebrities or public health authorities) suggests the vaccine carries risks, the resulting perception of vaccine risks can propagate quickly through the social network, fueling a

*alison.galvani@yale.edu. 
vaccine scare and a drop in vaccine coverage (biological contagion influences social contagion, see the figure, panel C).

In turn, the drop in vaccine coverage allows the number of individuals who are susceptible to infection to accumulate. When the percentage of susceptible individuals exceeds a tipping point, an outbreak of infectious disease occurs, which may motivate individuals to once again seek vaccination (social contagion influences biological contagion, see the figure, panel C). This dynamic may have occurred during whole cell pertussis vaccine scare in the United Kingdom during the 1970s and the measles-mumps-rubella vaccine autism scare more recently (4). Similarly, in some populations the advent of antiviral HIV drugs led to a rise in risky sexual behavior, and consequently a rise in sexually transmitted infections (5).

However, social contagions can also produce positive consequences in disease-behavior systems. Social norms dictate that individuals should cover their mouth when sneezing. Parents often vaccinate their children because other parents around them do so. Altruism can also be an important motivator (6). The Israeli Ministry of Health recently called for an additional dose of oral polio vaccine in children who were already vaccinated (and thus presumably protected), primarily to prevent infection in those who cannot get vaccinated due to weakened immune systems. Polio vaccine uptake soon exceeded the targets (7).

Epidemic trajectories and the uptake of control measures can vary widely between populations. SARS-CoV caused large epidemics in some populations but almost no transmission in others (8). Social differences between populations may be one reason for this. Control of SARS-CoV depended partly on population acceptance of quarantine and isolation, which is often determined by social norms. The role of disease-behavior interactions in outbreak heterogeneity has received little attention because of the difficulty of quantifying social feedbacks, but exploiting new sources of data such as online social media may help to address this (9).

The challenges of studying disease-behavior systems are generating interesting science. Mathematical modelers are exploring disease-behavior interactions not only in the context of vaccines but also for the emergence of antibiotic resistance and antiviral influenza drug resistance, as well as for social distancing, where people avoid contact with infected individuals (10). Interest in behavior-disease interactions in the context of HIV vaccines and antiviral drugs is also returning after a spike of interest in the 1990s. Modelers are exploring how disease-behavior dynamics, such as population susceptibility to vaccine risk, vary with socioeconomic factors (11).

Mathematical modelers are constructing realistic models tailored to specific systems and public health research questions, and testing the empirical validity of those models $(4,6$, 12). As part of this trend, increasing amounts of data on social contact networks are being collected in order to help formulate and test network-based disease-behavior models $(9,13)$. Moreover, modelers are incorporating the insights of economists, sociologists and psychologists into disease-behavior models (4, 6, 10-13).

Empirically validated disease-behavior models could be used, for example, as predictive tools to explore optimal strategies for public health intervention in response to a vaccine 
scare. Predicting "the madness of men" in disease-behavior systems may appear daunting. However, if social contagions have clear similarities to well-understood biological contagions that can be captured in models, and if the focus is on predicting aggregate population behavior rather than individual actions, predictive models may be feasible. Even an approximate ability to anticipate how populations will respond to new disease control measures could be helpful.

Predictability in such systems appears to be possible. For example, some models predicted that cervical cancer vaccine uptake would be below recommended levels in the United States, which is what ended up occurring $(12,15)$. Other models have retrospectively predicted vaccine coverage and endemic disease dynamics for historical pediatric vaccine scares (4). Empirical validation of models could be facilitated by more long-term data on the determinants of control measure uptake in the specific context of disease-behavior systems. Also, the field must develop a common lexicon. For example, social contagion is often referred to differently even when the underlying mathematical formulation is identical. This can create confusion. We have much to learn from those who "calculate the movement of stars"-astronomers routinely agree to standardize their nomenclature through committees.

Vaccines and drugs for many long-established infectious diseases are becoming more widely available. Thus, population behavior may replace accessibility as the most challenging barrier to higher uptake of control measures. On the other hand, for many novel zoonoses like SARS-CoV, there are initially no pharmaceutical interventions. Hence, public health must rely on quarantine, movement restrictions, and other measures that require cooperative behavior. Also, where there is lack of epidemiological knowledge in the early stages of a new zoonosis, fear and supposition can easily rush in to fill the void. In all cases, an improved understanding of the interplay between social contagions and biological contagions will help to improve disease control efforts.

\section{Acknowledgments}

CTB acknowledges grant support from NSERC, MEDI, CIHR and CFI. APG acknowledges grant support from NIH U01 GM15627, NSFSES-1227390, MIDAS U01 GM087719 and McDonnell Foundation 1220020114. The authors are grateful to Dan Yamin, Katie Atkins, David Durham, Angie Hofman, Scott Greenhalgh and Madhur Anand for helpful comments.

\section{References}

1. Christakis N, Fowler J. N Engl J Med. 2007; 357:370. [PubMed: 17652652]

2. May R, Levin S, Sugihara G. Nature. 2008; 451:893. [PubMed: 18288170]

3. O'Farrell, J. An Utterly Impartial History of Britain - Or 2000 Years of Upper Class Idiots In Charge. Doubleday; London: 2007.

4. Bauch CT, Bhattacharyya S. PLoS Comput Biol. 2012; 8:e1002452. [PubMed: 22496631]

5. Crepaz N, Hart T, Marks G. J Am Med Soc. 2004; 292:224.

6. Liu J, Kochin B, Tekle Y, Galvani A. J R Soc Interface. 2012; 9:68. [PubMed: 21632611]

7. Israeli Ministry of Health. [Access Date: 1 September 2013] http://www.health.gov.il/ NewsAndEvents/SpokemanMesseges/Pages/27082013_2.aspx

8. WHO. [Access Date: 1 September 2013.] Epidemic curves--severe acute respiratory syndrome (SARS). 2013. http://www.who.int/csr/sars/epicurve/epiindex/en/ 
9. Salathe M, Freifeld CC, Mekaru SR, Tomasulo AF, Brownstein JS. N Engl J Med. 2013; 369:401. [PubMed: 23822655]

10. Reluga TC. PLOS Comput Biol. 2010; 6:e1000793. [PubMed: 20523740]

11. d'Onofrio A, Manfredit P, Poletti P. J Theor Biol. 2011; 273:63. [PubMed: 21187103]

12. Basu S, Chapman G, Galvani A. Proc Natl Acad Sci USA. 2008; 105:19018. [PubMed: 19015536]

13. Funk S, Gilad E, Watkins C, Jansen VAA. Proc Natl Acad Sci USA. 2009; 106:6872. [PubMed: 19332788]

14. Kahan DM. Science. 2013 X, Y.

15. Williams WW, Lu P-J, Greby S, et al. MMWR. 2013; 62:66. [PubMed: 23364272] 

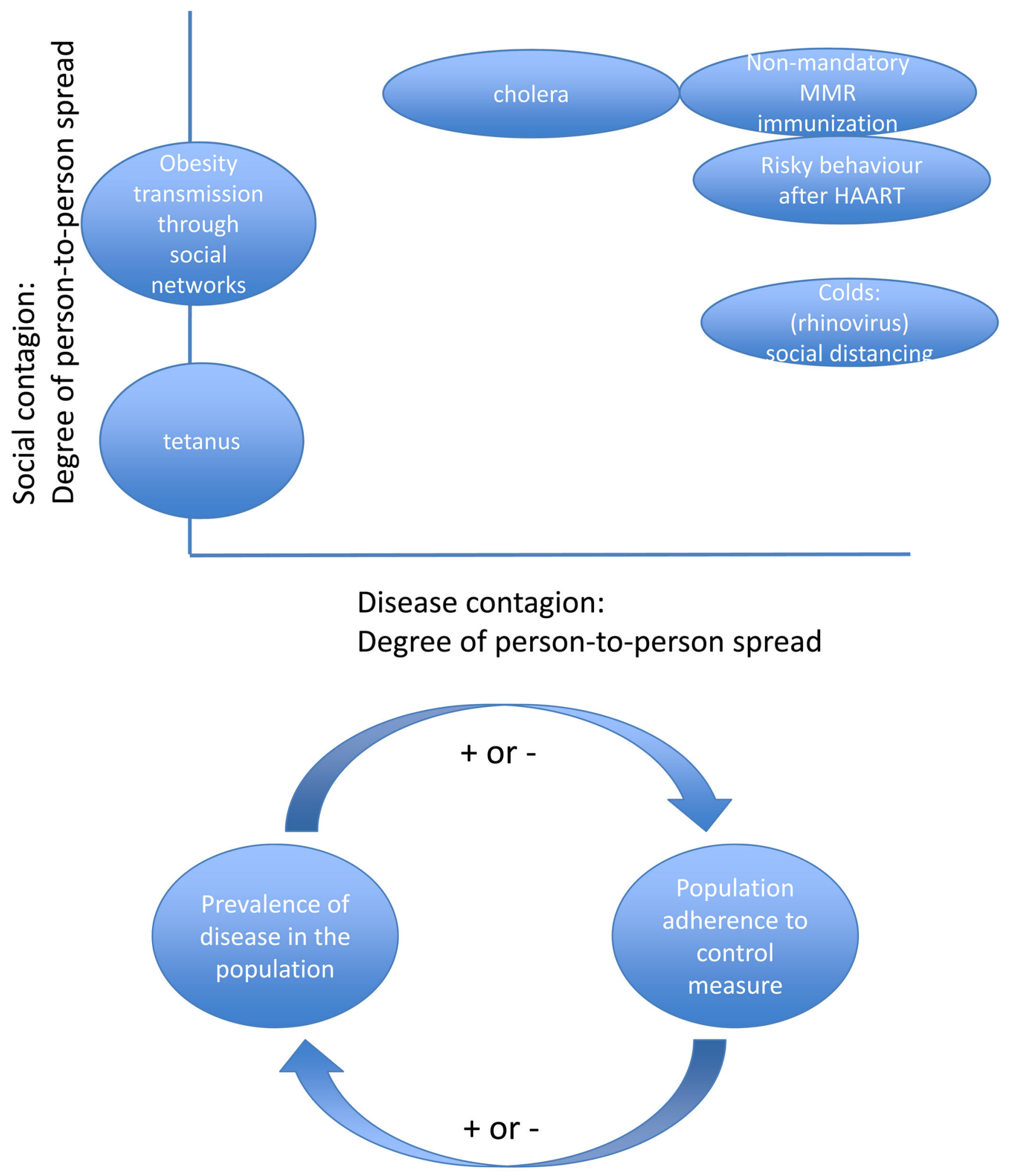

Figure 1. The whole is more than the sum of its parts in disease-behavior interactions When a social contagion on a social network (A) and a biological contagion on an infection contact network $(\mathbf{B})$ are coupled to one another $(\mathbf{C})$, the resulting interplay can create 
complex dynamics that are not present in either network layer in isolation, such as the fall and rise of vaccine coverage during a vaccine scare (4). Each person 1, 2, 3, 4... is a node in both the social contagion network and the biological contagion network. 